\title{
How do climbing fibers teach?
}

\section{Thomas S. Otis *, Paul J. Mathews, Ka Hung Lee and Jaione Maiz}

Department of Neurobiology and Center for Learning and Memory, Geffen School of Medicine at UCLA, Los Angeles, CA, USA

*Correspondence: otist@ucla.edu

Edited by:

Chris I De Zeeuw, Erasmus Medical Center Rotterdam, Netherlands

\section{A commentary on}

\section{A theory of cerebellar cortex}

by Marr, D. (1969). J. Physiol. 202, 437-470.

A theory of cerebellar function

by Albus, J. S. (1971). Math. Biosci. 10, 25-61.

Four decades ago, Marr and Albus suggested that the climbing fiber (CF) pathway from the inferior olive (IO) to the cerebellum instructs the cellular changes necessary for motor learning (Marr, 1969; Albus, 1971). Subsequent work has confirmed that CFs can drive specific forms of associative motor learning (Gilbert and Thach, 1977; Mauk et al., 1986; Raymond et al., 1996; Jirenhed et al., 2007; Medina and Lisberger, 2008) and has detailed how CFs trigger learning-related forms of synaptic plasticity in Purkinje neurons (PNs) (Linden et al., 1991; Linden and Connor, 1995; Coesmans et al., 2004). Yet, it is widely believed that associative motor learning, such as eyeblink conditioning (Lavond and Steinmetz, 1989; Perrett et al., 1993; Medina and Mauk, 1999; Jorntell and Ekerot, 2002; Ohyama et al., 2006; Shutoh et al., 2006) and vestibulo-ocular reflex (VOR) adaptation (Miles and Lisberger, 1981; Boyden et al., 2004) result from distinct forms of synaptic plasticity that are coordinated at multiple sites within the cerebellar circuit, an idea formalized in several reviews (Raymond et al., 1996; Boyden et al., 2004; Gao et al., 2012). This raises a central question regarding how CFs coordinates plasticity at multiple sites. Simply stated, how do CFs teach?

To address this larger question it is useful to focus on three previously hypothesized sites of associative synaptic plasticity within the cerebellar circuit. At each, the $\mathrm{CF}$ is believed to instruct heterosynaptic forms of plasticity by driving changes in the strengths of other excitatory inputs, however, the direction of change triggered by CF activity, i.e., long-term depression (LTD) or long-term potentiation (LTP), is different at each site (Figure 1A). The best described example of CF teaching, and the main focus of the Marr/Albus hypothesis, occurs at the parallel fiber (PF)-to-PN synapse. In mature PNs the single CF input generates a salient and distinctive signal-a cell wide burst termed the complex spike (Eccles et al., 1964) which instructs heterosynaptic LTD in those PFs that are coactivated with CFs [blue starburst, Figure 1A; (Wang et al., 2000; Hansel et al., 2001; Coesmans et al., 2004; Safo and Regehr, 2008)]. Albus also conjectured that CFs could drive plasticity at a second site, proposing heterosynaptic LTP of PF inputs to a subset of molecular layer interneurons [MLIs; red starburst in cortex, Figure 1A; (Albus, 1971)]. From the perspective of the PN, Albus considered CF enhancement of PF-to-MLI synapses as equivalent to "negative PF synaptic weights." Considering the fact that PNs spontaneously pacemake at rates up to $\sim 80 \mathrm{~Hz}$ (Hausser and Clark, 1997; Raman et al., 1997), PF-to-MLI LTP makes it possible to instruct learned pauses in $\mathrm{PN}$ spiking, something that PF LTD on its own cannot accomplish. Although this form of associative plasticity has yet to be demonstrated, there is evocative in vivo evidence that indirectly supports it (Jorntell and Ekerot, 2002). The final site at which CFs might instruct plasticity is at mossy fiber (MF)-to-deep cerebellar nucleus neuron (DCN) synapses (red starburst in the deep nuclei, Figure 1A). Much theoretical and experimental work supports the notion that MF-to-DCN synapses strengthen during associative learning (Miles and Lisberger, 1981; Lavond and Steinmetz, 1989; Perrett et al., 1993; Chen et al., 1996; Raymond et al., 1996; Garcia and Mauk, 1998; Medina and Mauk, 1999;
Ohyama et al., 2006; Shutoh et al., 2006), and some of these studies indicate that plasticity in the cortex may precede or consolidate plasticity in the DCN (Ohyama et al., 2006; Shutoh et al., 2006; Wulff et al., 2009). The predicted consequence of all three forms of plasticity is to increase DCN excitability in response to particular patterns of MF/PF inputs. While it is generally accepted that CFs drive associative LTD of PFs, it is not clear whether CFs drive the associative forms of LTP during learning (i.e., PF LTP at the MLIs and MF LTP at the DCN). Perhaps relatedly, there is also some debate to whether any one of these forms of plasticity, including PF LTD (Schonewille et al., 2011), are necessary for motor learning.

In an effort to better understand the mechanistic details of how CFs participate in cerebellar learning, we have exploited optogenetic and pharmacological approaches to selectively manipulate CF signals. Using adeno-associated viral delivery of ChR2-eYFP to IO neurons we are able to transfect CFs with high efficiency and specificity in the rat (Figure 1B; Mathews et al., 2012). Optical activation then gives rise to "pure" CF signals generated at the key sites within the cerebellar circuit identified in Figure 1A. This approach shows that MLIs are cooperatively excited by several CFs, giving rise to a robust, CF-driven, feed-forward inhibition that can in turn lead to a transient, synchronous pause in multiple PNs (dashed line, Figure 1A). The CF excitation of MLIs shows cooperativity in part because it can result from the indirect spillover of glutamate from multiple CFs to an MLI, a phenomenon first described by Barbour and colleagues (Szapiro and Barbour, 2007). In this way our observations suggest that MLIs might read out population activity in many CFs (Bell and Kawasaki, 1972; Welsh et al., 1995; Lang et al., 1999; Marshall and Lang, 2009; 

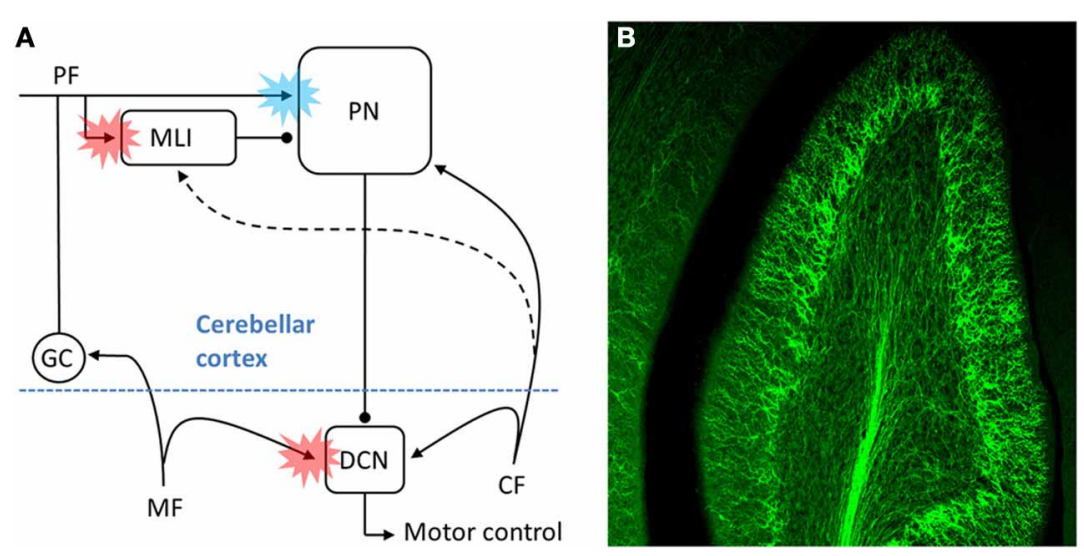

FIGURE 1 | The CF pathway and associative motor learning.

(A) Schematic of the proposed sites (colored bursts) within the cerebellar circuit where CFs might teach during associative motor learning. The blue burst denotes CF driven LTD of PFs, while the red bursts denote CF-driven LTP of either PF inputs (at MLI) or inputs MF (at DCN). As discussed in the text, for at least two of the three sites the physiological details of the CF input, the underlying cellular mechanisms of plasticity, and the relative contribution to behavioral learning remain to be elucidated. (B) ChR2-eYFP expressing CFs are visible in this multi-photon microscopic image of part of folia $\mathrm{X}$ in a $300 \mu \mathrm{m}$ thick rat brain slice.
Mukamel et al., 2009; Ozden et al., 2009), and that synchronous CF input to modules of PNs would result in synchronous pauses in PN spiking. In such a model, $\mathrm{CF}$-dependent pauses in groups of PNs could then serve as proxy teaching signals in the DCN.

In complimentary experiments we have used specific pharmacological tools to manipulate a component of the CF signal, the post-complex spike pause in simple spike firing rate. Two very different compounds (1-EBIO, a positive modulator of calcium activated $\mathrm{K}^{+}$channels, or ZD 7288, an inhibitor of hyperpolarization activated cation channels) were each demonstrated to prolong the postcomplex spike pause (Maiz et al., 2012). Either of these drugs infused into the cerebellar cortex during eyeblink conditioning resulted in markedly faster learning (Maiz et al., 2012). We hypothesize that prolongation of the $\mathrm{CF}$-associated pause drives faster learning by facilitating associative LTP of MF inputs to DCN neurons (see Figure 1A). Considering the NMDA receptor dependence of MF to DCN plasticity, it is straightforward to imagine how a pause in descending $\mathrm{PN}$ inhibition could associatively drive the types of LTP that have been described in vitro (Pugh and Raman, 2006, 2009).

A critical step in understanding cerebellar learning is to explain how CFs, or other teaching signals, coordinate learning-related changes within the circuit. The experiments described here address important questions about the biology of the CF and how it might operate as a teaching signal. Related questions include whether CFs give rise to distinctive postsynaptic signals at those sites in the circuit where they have been hypothesized as teachers, and whether CFs trigger heterosynaptic, associative forms of plasticity that might contribute to learned motor behaviors. The striking anatomical organization of the cerebellar cortex coupled with the remarkable properties of the CF contact on PNs led to the insightful conjecture of Marr and Albus more than 40 years ago. Our observations are consistent with CFs exerting control over multiple sites within the cerebellar circuit, in part through indirect actions read out by MLIs or groups of PNs, a picture that brings to mind the aphorism from the Talmud, "When you teach your son, you teach your son's son." Future experiments utilizing a wide breadth of classical and novel techniques, like those mentioned here will be required to determine just how paternalistic the CF is, and whether it broadens its influence in an analogous way.

\section{REFERENCES}

Albus, J. S. (1971). A theory of cerebellar function. Math. Biosci. 10, 25-61.

Bell, C. C., and Kawasaki, T. (1972). Relations among climbing fiber responses of nearby Purkinje Cells. J. Neurophysiol. 35, 155-169.

Boyden, E. S., Katoh, A., and Raymond, J. L. (2004). Cerebellum-dependent learning: the role of multiple plasticity mechanisms. Annu. Rev. Neurosci. 27, 581-609.

Chen, L., Bao, S., Lockard, J. M., Kim, J. K., and Thompson, R. F. (1996). Impaired classical eyeblink conditioning in cerebellar-lesioned and Purkinje cell degeneration (pcd) mutant mice. J. Neurosci. 16, 2829-2838.

Coesmans, M., Weber, J. T., De Zeeuw, C. I., and Hansel, C. (2004). Bidirectional parallel fiber plasticity in the cerebellum under climbing fiber control. Neuron 44, 691-700.

Eccles, J., Llinas, R., and Sasaki, K. (1964). Excitation of cerebellar Purkinje cells by the climbing fibres. Nature 203, 245-246.

Gao, Z., van Beugen, B. J., and De Zeeuw, C. I. (2012). Distributed synergistic plasticity and cerebellar learning. Nat. Rev. Neurosci. 13, 619-635.

Garcia, K. S., and Mauk, M. D. (1998). Pharmacological analysis of cerebellar contributions to the timing and expression of conditioned eyelid responses. Neuropharmacology 37, 471-480.

Gilbert, P. F., and Thach, W. T. (1977). Purkinje cell activity during motor learning. Brain Res. 128, 309-328.

Hansel, C., Linden, D. J., and D’Angelo, E. (2001). Beyond parallel fiber LTD: the diversity of synaptic and non-synaptic plasticity in the cerebellum. Nat. Neurosci. 4, 467-475.

Hausser, M., and Clark, B. A. (1997). Tonic synaptic inhibition modulates neuronal output pattern and spatiotemporal synaptic integration. Neuron $19,665-678$.

Jirenhed, D. A., Bengtsson, F., and Hesslow, G. (2007). Acquisition, extinction, and reacquisition of a cerebellar cortical memory trace. J. Neurosci. 27, 2493-2502.

Jorntell, H., and Ekerot, C. F. (2002). Reciprocal bidirectional plasticity of parallel fiber receptive fields in cerebellar Purkinje cells and their afferent interneurons. Neuron 34, 797-806.

Lang, E. J., Sugihara, I., Welsh, J. P., and Llinas, R. (1999). Patterns of spontaneous purkinje cell 
complex spike activity in the awake rat. J. Neurosci. 19, 2728-2739.

Lavond, D. G., and Steinmetz, J. E. (1989). Acquisition of classical conditioning without cerebellar cortex. Behav. Brain Res. 33, 113-164.

Linden, D. J., and Connor, J. A. (1995). Longterm synaptic depression. Annu. Rev. Neurosci. 18, 319-357.

Linden, D. J., Dickinson, M. H., Smeyne, M., and Connor, J. A. (1991). A long-term depression of AMPA currents in cultured cerebellar Purkinje neurons. Neuron 7, 81-89.

Maiz, J., Karakossian, M. H., Pakaprot, N., Robleto, K., Thompson, R. F., and Otis, T. S. (2012). Prolonging the postcomplex spike pause speeds eyeblink conditioning. Proc. Natl. Acad. Sci. U.S.A. 109, 16726-16730.

Marr, D. (1969). A theory of cerebellar cortex. J. Physiol. 202, 437-470.

Marshall, S. P., and Lang, E. J. (2009). Local changes in the excitability of the cerebellar cortex produce spatially restricted changes in complex spike synchrony. J. Neurosci. 29, 14352-14362.

Mathews, P. J., Lee, K. H., Peng, Z., Houser, C. R., and Otis, T. S. (2012). Effects of climbing fiber driven inhibition on Purkinje neuron spiking. J. Neurosci. 32 , (in press).

Mauk, M. D., Steinmetz, J. E., and Thompson, R. F. (1986). Classical conditioning using stimulation of the inferior olive as the unconditioned stimulus. Proc. Natl. Acad. Sci. U.S.A. 83, 5349-5353.

Medina, J. F., and Lisberger, S. G. (2008). Links from complex spikes to local plasticity and motor learning in the cerebellum of awake-behaving monkeys. Nat. Neurosci. 11, 1185-1192.

Medina, J. F., and Mauk, M. D. (1999). Simulations of cerebellar motor learning: computational analysis of plasticity at the mossy fiber to deep nucleus synapse. J. Neurosci. 19, 7140-7151.

Miles, F. A., and Lisberger, S. G. (1981). Plasticity in the vestibulo-ocular reflex: a new hypothesis. Annu. Rev. Neurosci. 4, 273-299.

Mukamel, E. A., Nimmerjahn, A., and Schnitzer, M. J. (2009). Automated analysis of cellular signals from large-scale calcium imaging data. Neuron 63 , 747-760.

Ohyama, T., Nores, W. L., Medina, J. F., Riusech, F. A. and Mauk, M. D. (2006). Learning-induced plasticity in deep cerebellar nucleus. J. Neurosci. 26, 12656-12663.

Ozden, I., Sullivan, M. R., Lee, H. M., and Wang, S. S. (2009). Reliable coding emerges from coactivation of climbing fibers in microbands of cerebellar Purkinje neurons. J. Neurosci. 29, 10463-10473.

Perrett, S. P., Ruiz, B. P., and Mauk, M. D. (1993). Cerebellar cortex lesions disrupt learningdependent timing of conditioned eyelid responses. J. Neurosci. 13, 1708-1718.

Pugh, J. R., and Raman, I. M. (2006). Potentiation of mossy fiber EPSCs in the cerebellar nuclei by NMDA receptor activation followed by postinhibitory rebound current. Neuron 51, 113-123.

Pugh, J. R., and Raman, I. M. (2009). Nothing can be coincidence: synaptic inhibition and plasticity in the cerebellar nuclei. Trends Neurosci. 32, 170-177.

Raman, I. M., Sprunger, L. K., Meisler, M. H., and Bean, B. P. (1997). Altered subthreshold sodium currents and disrupted firing patterns in Purkinje neurons of Scn8a mutant mice. Neuron 19, 881-891.

Raymond, J. L., Lisberger, S. G., and Mauk, M. D. (1996). The cerebellum: a neuronal learning machine? Science 272, 1126-1131.

Safo, P., and Regehr, W. G. (2008). Timing dependence of the induction of cerebellar LTD. Neuropharmacology 54, 213-218.
Schonewille, M., Gao, Z., Boele, H. J., Veloz, M. F., Amerika, W. E., Simek, A. A., et al. (2011). Reevaluating the role of LTD in cerebellar motor learning. Neuron 70, 43-50.

Shutoh, F., Ohki, M., Kitazawa, H., Itohara, S., and Nagao, S. (2006). Memory trace of motor learning shifts transsynaptically from cerebellar cortex to nuclei for consolidation. Neuroscience 139, 767-777.

Szapiro, G., and Barbour, B. (2007). Multiple climbing fibers signal to molecular layer interneurons exclusively via glutamate spillover. Nat. Neurosci. 10, 735-742.

Wang, S. S., Denk, W., and Hausser, M. (2000). Coincidence detection in single dendritic spines mediated by calcium release. Nat. Neurosci. 3, 1266-1273.

Welsh, J. P., Lang, E. J., Suglhara, I., and Llinas, R. (1995). Dynamic organization of motor control within the olivocerebellar system. Nature 374, 453-457.

Wulff, P., Schonewille, M., Renzi, M., Viltono, L., Sassoè-Pognetto, M., Badura, A., et al. (2009). Synaptic inhibition of Purkinje cells mediates consolidation of vestibulo-cerebellar motor learning. Nat. Neurosci. 12, 1042-1049.

Received: 16 September 2012; accepted: 11 November 2012; published online: 30 November 2012.

Citation: Otis TS, Mathews PJ, Lee KH and Maiz J (2012) How do climbing fibers teach? Front. Neural Circuits 6:95. doi: 10.3389/fncir.2012.00095

Copyright (๑) 2012 Otis, Mathews, Lee and Maiz. This is an open-access article distributed under the terms of the Creative Commons Attribution License, which permits use, distribution and reproduction in other forums, provided the original authors and source are credited and subject to any copyright notices concerning any third-party graphics etc. 